\title{
The Pattern of Lumbosacral List Curve in Patients with Lumbar Disc
} Herniation

\author{
Hasan Ghandhari ${ }^{1}$, Farshad Nikouei ${ }^{1}$, Saeed Sabbaghan ${ }^{1}$, Maryam Ameri Mahabadi ${ }^{2}$, Mona Ziaei ${ }^{3}$, \\ Behrooz Givehchian ${ }^{1, *}$ and Farshad Safdari ${ }^{4}$ \\ ${ }^{1}$ Bone and Joint Reconstruction Research Center, Shafa Orthopedic Hospital, Iran University of Medical Sciences, Tehran, Iran \\ ${ }^{2}$ Forensic and Legal Medicine Specialist, Iran University of Medical Sciences, Tehran, Iran \\ ${ }^{3} \mathrm{MD}$, Tehran, Iran \\ ${ }^{4}$ Bone Joint and Related Tissues Research Center, Shahid Beheshti University of Medical Sciences, Tehran, Iran \\ "Corresponding author: Bone and Joint Reconstruction Research Center, Shafa Orthopedic Hospital, Iran University of Medical Sciences, Tehran, Iran. Email: \\ b.givehchian.spine@gmail.com
}

Received 2018 December 12; Revised 2019 January 27; Accepted 2019 February 08.

\begin{abstract}
Background: There are many unanswered questions about the characteristics and mechanism of the lumbosacral scoliotic list (LSL). In the current study, the pattern of LSL, the level of maximal opened disc space (take-off) in addition to the relationship between the location of disc herniation (DH) on magnetic resonance imaging (MRI) and LSL direction on radiographs, were investigated. Methods: A total of 37 patients, with extruded lumbar DH and LSL, were included in the current study. The following variables were measured on standing anteroposterior and lateral lumbar x-rays: LSL (from L1 to L5), the take-off level, and the coronal shift (the distance between the plumb line from T12 spinous process to the central sacral vertical line). The direction of LSL was recorded as the bending side of the patient opposite to the convexity of the curve. The location of DH was determined as right, left, or central on an MRI.

Results: The magnitude of the LSL curve averaged $9.9^{\circ} \pm 6.9^{\circ}$. Regarding the right or left herniation, the list occurred mostly toward the opposite side of the herniation direction $(\mathrm{P}=0.04)$. There was no significant matching between the location of herniation and the take-off segment $(\mathrm{P}=0.391)$; however, in $67.6 \%$ of patients with L4 - L5 or L5-S1 herniation, the take-off point occurred one segment above the involved levels. The take-off was found only at L3 - L4 or L4 - L5 levels in all the patients.

Conclusions: LSL usually occurs on the opposite side of the herniation location. Furthermore, take-off is found in L3 - L4 or L4 - L5 segments in most of the patients with LSL. It seems that LSL curve characters are not affected by the level of herniation.
\end{abstract}

Keywords: Disc Herniation, Lumbar Spine, Lumbosacral List, Coronal Shift

\section{Background}

Lumbosacral scoliotic list (LSL) is a well-known indicator of lumbar disc herniation (DH) $(1,2)$. There are several terms used to describe the condition such as trunk list (3), sciatic scoliosis list (4), sciatic spinal deformity (5), and lumbosacral scoliotic list (6). This lateral bending is transient and evident upon physical examination (7). In other words, LSL is a non-structural scoliosis resulted from irritating a nerve root.

The mechanism of the list is controversial. Finneson described the condition, thus: when a disc is herniated lateral to the nerve root, the patient leans the body to the contralateral side to decompress the root, and vice versa occurs when the herniation occurs medial to the root, the patient bends toward the root to decrease nerve tension (4). However, some studies did not confirm the hypothesis and stated that there is no correlation between the anatomic location of DH and the side of LSL $(1,4-6)$. It has been shown that nerve root pressure is higher in patients with LSL than patients without a list; however, this is not the sole mechanism of this phenomenon (8). Few studies have shown that $\mathrm{DH}$ is more common in the convex side of the list while the trunk shifts to the contralateral side $(4,6,9,10)$.

These controversial findings may have resulted from different study designs and evaluation methods. Previous investigations of LSL were based on clinical features and surgical findings.

\section{Objectives}

The purpose of this study was to investigate the pattern and level of maximal bending of the lumbar spine (take-off) in LSL. In addition, the radiographic relationship 
between DH location on the magnetic resonance imaging (MRI) and LSL direction on plain radiography was evaluated.

\section{Methods}

Between September 2015 and August 2017, patients with extruded lumbar DH and LSL, who were candidates for surgical treatment, enrolled in this prospective study. Before the study, the Institutional Review Board approved the protocol, and eligible patients signed informed consent. The indications of surgical intervention consisted of a nonresponsive pain to conservative treatment for six weeks, progressive neurologic deficit, or cauda equina syndrome. Patients with more than one level of $\mathrm{DH}$, degenerative disc disease in the lumbar spine, Modic changes in MRI, history of spinal deformity, and previous spinal surgery were excluded from this study.

At first, on the physical examination, the side of patient bending was recorded. Then, the standing lumbosacral $\mathrm{X}$-rays in anteroposterior and lateral views were taken, and lumbosacral MRI was obtained (1.5 T) (Figure 1). All of the radiographic measurements were performed using Surgimap $^{\mathrm{TM}}$, version 2.2.12.1 (Surgimap Spine Software, Nemaris Inc., New York, USA). Two investigators (one spine fellow and one spine surgeon) analyzed the images and measured the obtained values independently. The mean of the two measurements was considered as the final value. In a pilot study, the Cronbach's alpha was $>0.8$ for interobserver reliability of the two investigators.

The list angle measurement from the superior endplate of L1 to the inferior endplate of L5 (Figure 2) was car-

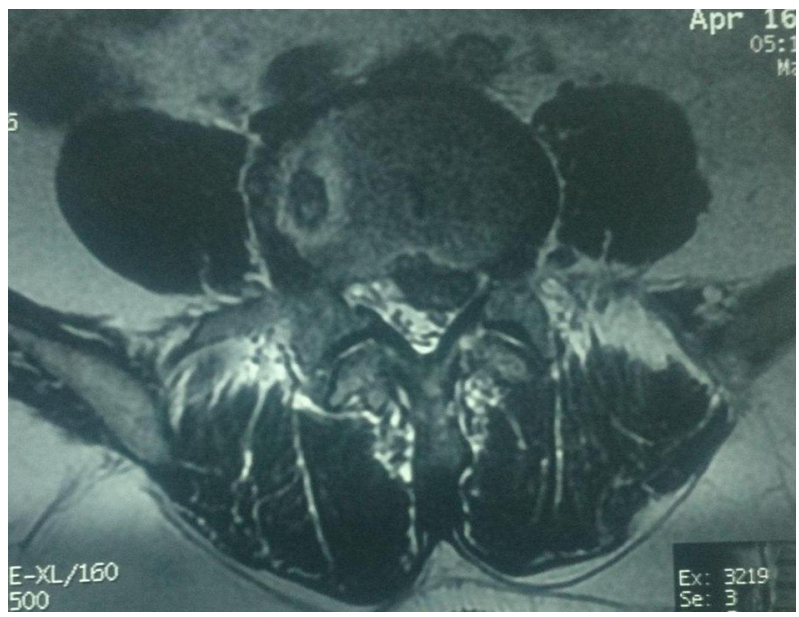

Figure 1. MRI T2-sequence showing L4 - L5 left side disc herniation in a 55-year old man. ried out by using Cobb's angle. In each disc space, the angle between the superior endplate of the caudal vertebra and the inferior endplate of the cephalad was measured (Figure 3). Whenever the angle opened to the convex side of the curve, the value was considered positive, and when the angle opened to the concave side it was called negative. When the value of a disc space angle in the upper segments was negative, which implies they were opened to the concave side of the LSL curve, the maximum LSL Cobb's angle was measured in the lower segments. The deviation to the left or right was also determined. In addition, the take-off level was defined as disc space with maximal Cobb's angle from L1 to S1 (Figure 3). The coronal shift was defined as the distance between the plumb line from the T12 spinous process to the central sacral vertical line (CSVL). Diagnosis of LSL was made based on the coronal shift. The direction of the list was considered as the bending side of the patient opposite to the convexity of the curve.

Finally, a spinal surgeon who was blinded to the X-rays independently analyzed the MRI sequences to locate $\mathrm{DH}$ as

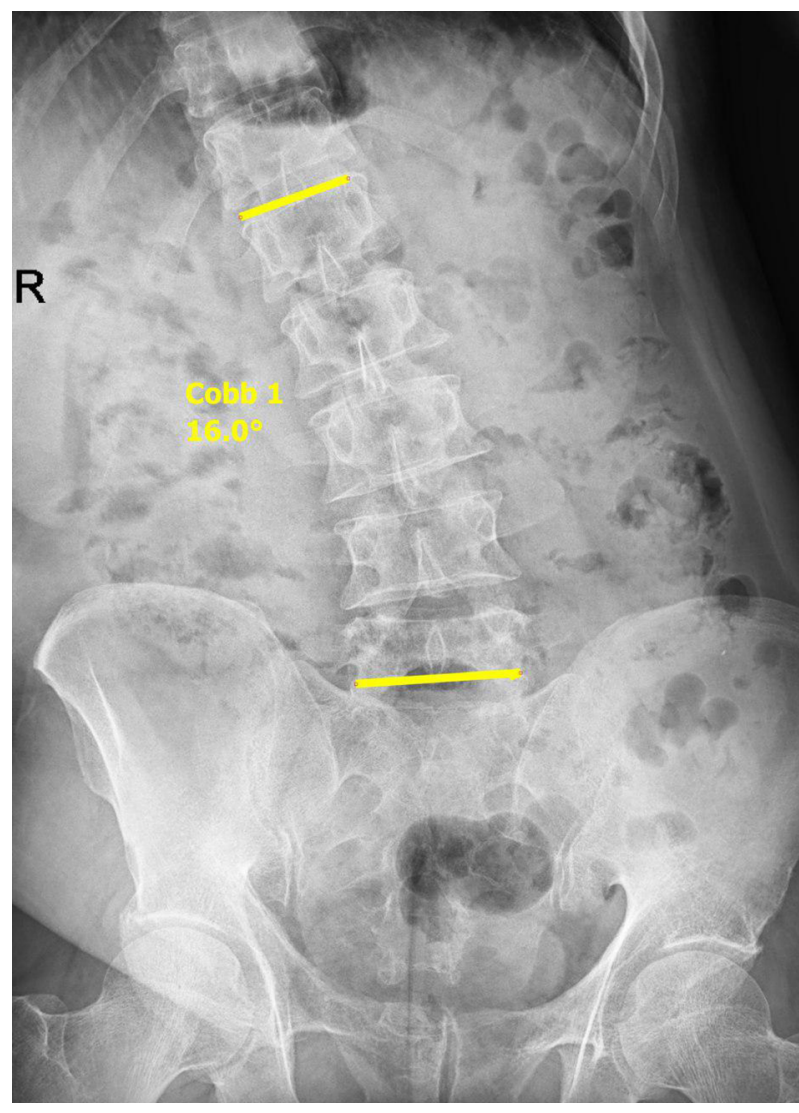

Figure 2. Lumbosacral radiography, AP view. Cobb's angle between superior endplate of L1 and inferior endplate of L5 recorded as lumbosacral list. 


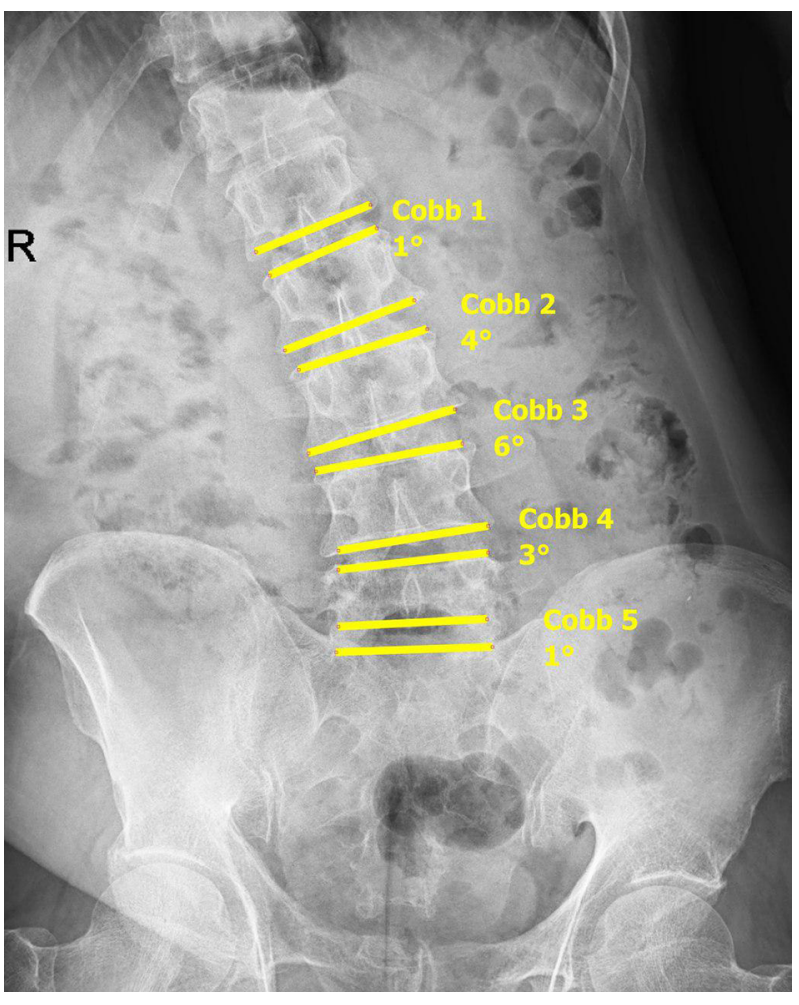

Figure 3. Lumbosacral radiography, AP view. Cobb's angle measurement in each disc space showing the take-off occurred in L3 - L4 $\left(6^{\circ}\right)$.

right, left, or central.

\subsection{Data Analyses}

Data were analyzed using SPSS statistical software V. 15.0. To compare the qualitative data, chi-square test was used. Pearson's correlation test was used to investigate the correlation between LSL and coronal shift. Cronbach's alpha was calculated to measure interobserver reliability. P $<0.05$ was considered as a significant finding.

\section{Results}

The characteristics of the patients are presented in Table 1. A total of 37 patients were enrolled in the current study. The mean of LSL curve magnitude measured from L1 superior endplate to L5 inferior endplate was $9.9^{\circ} \pm 6.9^{\circ}$ (range: $1^{\circ}$ to $29^{\circ}$ ). Furthermore, the mean of maximum LSL was $11.4^{\circ} \pm 6^{\circ}\left(3^{\circ}\right.$ to $\left.29^{\circ}\right)$. Generally, there was no statistically significant relationship between the list direction and the side of herniation $(\mathrm{P}=0.123)$ (Table 2). However, when central hernias were excluded, it was observed that the list direction was significantly related to the herniation

\begin{tabular}{|c|c|}
\hline Characteristics & Values \\
\hline No. & 37 \\
\hline \multicolumn{2}{|l|}{ Age, $y$} \\
\hline Mean \pm SD & 35.79 .5 \\
\hline Range & $18-56$ \\
\hline \multicolumn{2}{|l|}{ Gender } \\
\hline Male & $24(64.9)$ \\
\hline Female & $13(35.1)$ \\
\hline \multicolumn{2}{|c|}{ Level of disc herniation } \\
\hline L3 - L4 & $3(8.1)$ \\
\hline L4 - L5 & $22(59.5)$ \\
\hline L5 - S1 & $12(32.4)$ \\
\hline \multicolumn{2}{|c|}{ Location of disc herniation } \\
\hline Right & $15(40.5)$ \\
\hline Left & $12(32.5)$ \\
\hline Central & $10(27)$ \\
\hline \multicolumn{2}{|l|}{ LIST (degree) } \\
\hline Mean $\pm S D$ & $9.9 \pm 6.9$ \\
\hline Range & $1-29$ \\
\hline \multicolumn{2}{|c|}{ Maximal LIST (degree) } \\
\hline Mean $\pm S D$ & $11.4 \pm 6$ \\
\hline Range & $3-29$ \\
\hline \multicolumn{2}{|l|}{ List side } \\
\hline Right & $14(37.8)$ \\
\hline Left & $23(62.2)$ \\
\hline
\end{tabular}

${ }^{a}$ Values are expressed as No. (\%) unless otherwise indicated.

side $(\mathrm{P}=0.04)$ and occurred mostly toward the opposite side of the herniation (Table 2).

Local Cobb's angle in each lumbar disc was measured, which was maximal in the take-off level and decreasing toward cephalad or caudal (Figure 4). Of the three patients with L3 - L4 herniation, take-off was found at the same disc space in one case and at the lower segment in two other patients. In patients with $\mathrm{L} 4$ - $\mathrm{L} 5 \mathrm{DH}(\mathrm{n}=22)$, take-off occurred at one level above in 16 patients ( $72.7 \%)$, it took place at the same level in 11 patients. When the herniation was in L5 - S1 $(\mathrm{n}=12)$, take-off was found one level above in seven patients (58.3\%) and two levels above in five patients (41.7\%). Generally, in 34 patients with L4 - L5 and L5 - S1 hernias, take-off occurred one level above in $67.6 \%$. However, there was no statistically significant relationship between the level of herniation and take-off level $(\mathrm{P}=0.391)$ (Table 3$)$. In addition, take-off was found only at L3 - L4 or L4 - L5 levels in all the patients. 


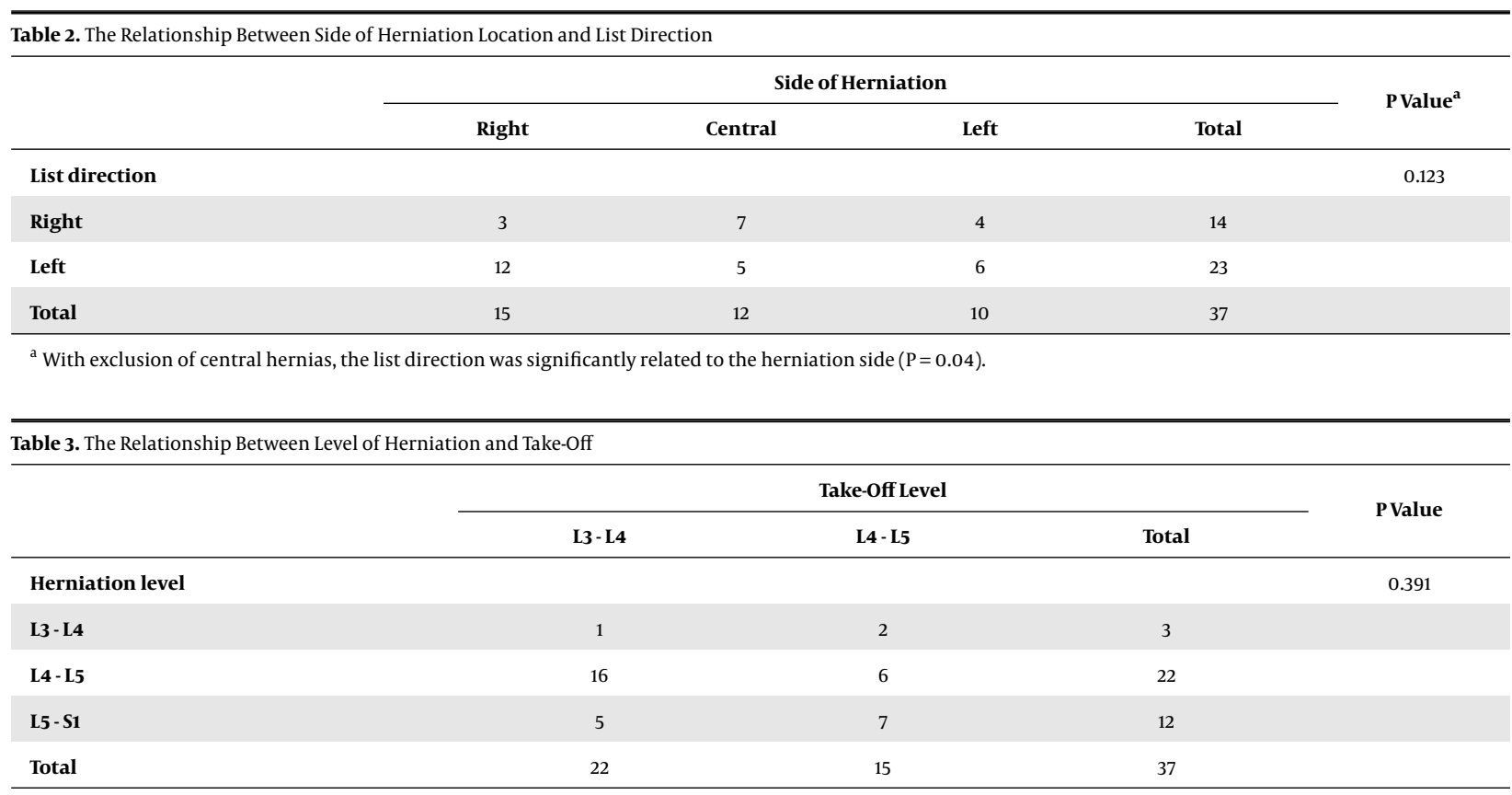

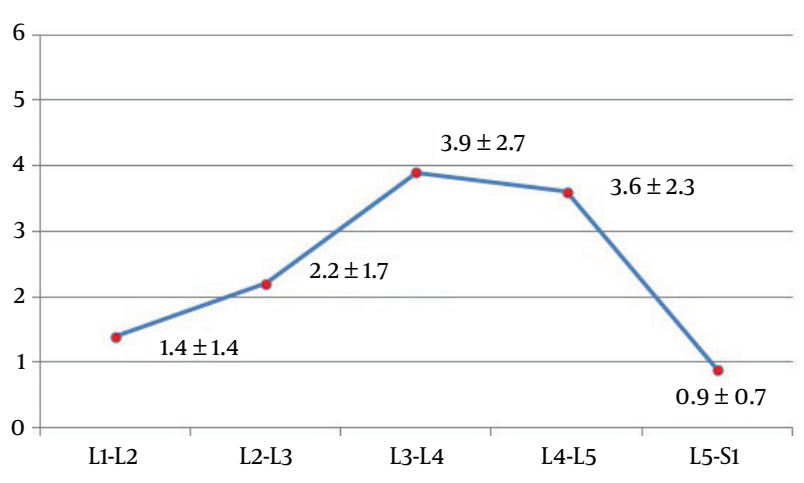

Figure 4. Lumbosacral radiography, AP view. The mean Cobb's angle in each lumbar disc space (degree).

The average coronal shift was $18.9 \pm 11.6 \mathrm{~mm}$ ( 4 to 62 $\mathrm{mm}$ ). Pearson's correlation test revealed that there was a significant positive correlation between LSL and coronal $\operatorname{shift}(\mathrm{r}=0.6, \mathrm{P}=0.001)$.

\section{Discussion}

Sudden onset of scoliosis with the presentation of concurrent lumbar and/or lower extremity pain is highly suggestive of a DH (11). The incidence of LSL has been reported to range between $8.9 \%$ to $18 \%$ in patients with lumbar $\mathrm{DH}$ who have undergone a discectomy $(4,10)$. However, there is a lack of knowledge regarding the causes and characteristics of this phenomenon. Matsui et al. (4), reported that these postural changes occur to protect the spinal nerves from further damage. The radiologic features of LSL are different from idiopathic scoliosis as LSL exhibits a short lumbosacral curve without vertebral rotation in the axial plane (9). Some previous studies have reported the disappearance of LSL after the treatment of causative factors (4, $6,7,10)$. Gillan et al. (3), reported that there was a poor correlation between LSL magnitudes and clinical outcomes.

The purpose of the current study was to determine the pattern of LSL in patients with DH. To this end, the site of herniation on an MRI was compared to the LSL direction on plain radiography. In paracentral DHs (right or left), LSL was more to the opposite side of herniation in most of the cases $(\mathrm{P}=0.04)$. In addition, when the disc was herniated centrally, no relationship to the LSL direction was determined.

In agreement with the current study, it has been reported that in more than $80 \%$ of patients with LSL, the trunk shifted away from the herniation side $(4,10,12,13)$. Suk et al. (6), evaluated the association between location (medial or lateral to the nerve root) and level of $\mathrm{DH}$ and the direction of LSL in 45 patients. The authors reported that there was no association between the anatomic location of DH and LSL direction. However, significantly, twothirds of the patients listed to the opposite side of the hernia (6). Matsui stated that when the lumbar spine bends to the lateral side, the intervertebral disc becomes wedgeshaped and the disc stretches and reduces in size, which Suk called: autonomic decompression $(4,6)$. In an article, Zhu et al. (9), reviewed studies on 26 adolescents with 
lumbar DH, and reported that the direction of lumbosacral curve and trunk shift was related to the side of $\mathrm{DH}$.

The results of our study showed that in all the patients with LSL, take-off occurred at L3 - L4 or L4 - L5 levels. When a take-off is found in L3 - L4 or L4 - L5 levels, there is a disc extrusion in $75 \%$ of the cases in L4 - L5 and L5 - S1 levels. This finding is interesting since most of the hernias are found at one of these two levels (96\%) (14).

Take-off did not occur in levels L5 - S1 in all patients. Kim et al. (10), reported that this was due to the fact that L5 - S1 level is enclosed within the pelvic cavity. In other words, it was found that the mean Cobb's angle increased gradually from L5 - S1 to L3 - L4 in each level and then decreased gradually to the L1 - L2. The best evidence that explains this phenomenon was reported by White and Panjabi. They demonstrated that the range of lateral bending in the lumbar spine increased from L5 - S1 $\left(3^{\circ}\right)$ to L3 - L4 $\left(8^{\circ}\right)$ and then decreases to L1 - L2 $\left(6^{\circ}\right)$, while the minimum range was found in L5 - S1 (15). Therefore, it seems that the LSL pattern is related to the biomechanics of spinal motion segments rather than DH level. The resulting coronal imbalance increased significantly with increased LSL magnitude. The greater the Cobb's angle, the greater the patient shift from CSVA.

One of the advantages of the current study was that axial MRI sequences were used to categorize the disc herniations as the central and right or left paracentral. These findings were useful to investigate the relationship between the herniation side and list direction, which was not investigated in the other studies $(4,6,7,10)$. In addition, we found that central disc herniation is not associated with a particular direction of LSL, which was not reported previously. Another advantage of the study was that the pattern of LSL was investigated based on Cobb's angle in each disc level and take-off level, which was not determined in previous studies.

Like any other study, some limitations were observed in the current study. The long cassette scoliotic plain radiography was not used to determine other curves in our patients. However, the use of long cassette produces more details regarding the pattern of compensatory curves in the thoracic spine. Clinical outcomes were not investigated in this study due to the fact that the study aimed to analyze the radiographic nature of LSL. There was no follow-up after surgery to find out when and how the LSL disappeared in the patients.

\subsection{Conclusions}

The findings of the current study showed that when the patient leans to one side as a result of disc herniation, there is usually an extruded disc on the opposite side. When LSL occurs, maximal bending (take-off) is found in L3
- L4 or L4 - L5 levels possibly due to the maximal range of lateral bending within these segments that naturally occurs. It appears that LSL pattern is not affected by the level of herniation. In addition, larger degrees of LSL make the patient more imbalanced in the coronal plane.

\section{Footnotes}

Authors' Contribution: Study concept and design: Hasan Ghandhari and Farshad Nikouei; acquisition of data: Farshad Nikouei, Hasan Ghandhari, Behrooz Givehchian, and Mona Ziaei; analysis and interpretation of data: Maryam Ameri Mahabadi and Farshad Safdari; drafting of the manuscript: Maryam Ameri Mahabadi, Farshad Safdari, and Mona Ziaei; critical revision of the manuscript for important intellectual content: Saeed Sabbaghan, Behrooz Givehchian, and Maryam Ameri Mahabadi; statistical analysis: Farshad Safdari; administrative, technical, and material support: Behrooz Givehchian, Saeed Sabbaghan, and Mona Ziaei; study supervision: Farshad Nikouei, Hasan Ghandhari, and Saeed Sabbaghan.

Conflict of Interests: There was no conflict of interests to declare.

Ethical Approval: The study was approved by the Ethical Committee of Bone and Joint Reconstruction Research Center, Shafa Orthopedic Hospital, Iran University of Medical Sciences.

Funding/Support: There was no funding on this study.

\section{References}

1. Porter RW, Miller CG. Back pain and trunk list. Spine (Phila Pa 1976). 1986;11(6):596-600. doi: 10.1097/00007632-198607000-00011. [PubMed: 2947332].

2. Harrison DE, Cailliet R, Betz JW, Harrison DD, Colloca CJ, Haas JW, et al. A non-randomized clinical control trial of Harrison mirror image methods for correcting trunk list (lateral translations of the thoracic cage) in patients with chronic low back pain. Eur Spine J. 2005;14(2):155-62. doi: 10.1007/s00586-004-0796-z. [PubMed: 15517424]. [PubMed Central: PMC3476700].

3. Gillan MG, Ross JC, McLean IP, Porter RW. The natural history of trunk list, its associated disability and the influence of McKenzie management. EurSpine J.1998;7(6):480-3. doi:10.1007/s005860050111. [PubMed: 9883957]. [PubMed Central: PMC3611301].

4. Matsui H, Ohmori K, Kanamori M, Ishihara H, Tsuji H. Significance of sciatic scoliotic list in operated patients with lumbar disc herniation. Spine (Phila Pa 1976). 1998;23(3):338-42. doi: 10.1097/00007632199802010-00010. [PubMed: 9507622].

5. Lorio MP, Bernstein AJ, Simmons EH. Sciatic spinal deformitylumbosacral list: An "unusual" presentation with review of the literature. Journal of Spinal Disorders. 1995;8(3):201-5. doi: 10.1097/00002517199506000-00004. [PubMed: 7670210].

6. Suk KS, Lee HM, Moon SH, Kim NH. Lumbosacral scoliotic list by lumbar disc herniation. Spine (Phila Pa 1976). 2001;26(6):667-71. doi: 10.1097/00007632-200103150-00023. [PubMed: 11246383]. 
7. Arangio GA, Hartzell SM, Reed JF. Significance of lumbosacral list and low-back pain. A controlled radiographic study. Spine (Phila Pa 1976). 1990;15(3):208-10. doi: 10.1097/00007632-199003000-00009. [PubMed: 2141185].

8. Takahashi K, Shima I, Porter RW. Nerve root pressure in lumbar disc herniation. Spine (Phila Pa 1976). 1999;24(19):2003-6. doi: 10.1097/00007632-199910010-00007. [PubMed:10528375].

9. Zhu Z, Zhao Q, Wang B, Yu Y, Qian B, Ding Y, et al. Scoliotic posture as the initial symptom in adolescents with lumbar disc herniation: Its curve pattern and natural history after lumbar discectomy. BMC Musculoskelet Disord. 2011;12:216. doi: 10.1186/1471-2474-12-216. [PubMed: 21962233]. [PubMed Central: PMC3196737].

10. Kim R, Kim RH, Kim CH, Choi Y, Hong HS, Park SB, et al. The incidence and risk factors for lumbar or sciatic scoliosis in lumbar disc herniation and the outcomes after percutaneous endoscopic discectomy.
Pain Physician. 2015;18(6):555-64. [PubMed: 26606007].

11. Hsu KL, Tai TW, Chien JT, Lin CJ. Sciatic scoliosis: An easily misdiagnosed disease in adolescents and young adults. Tzu Chi Medical Journal. 2012;24(4):196-200. doi:10.1016/j.tcmj.2012.10.006.

12. Kleinberg S. Sciatic scoliosis. Am J Surg. 1950;80(3):332-7. doi: 10.1016/0002-9610(50)90507-1. [PubMed: 15432831].

13. Finneson BE. Low back pain. Philadelphia: J. B. Lippincott Company; 1973.

14. Hao DJ, Duan K, Liu TJ, Liu JJ, Wang WT. Development and clinical application of grading and classification criteria of lumbar disc herniation. Medicine (Baltimore). 2017;96(47). e8676. doi: 10.1097/MD.0000000000008676. [PubMed: 29381945]. [PubMed Central: PMC5708944]

15. White AA, Panjabi MM. Clinical biomechanics of the spine. 2nd ed. Philadelphia: JB Lippincott; 1999. 